\title{
The impact of a mobile COVID-19 polymerase chain reaction laboratory at a large tertiary hospital during the first wave of the pandemic: A retrospective analysis
}

\author{
S Omar, ${ }^{1}$ MB ChB, FC Path (SA) Chem, DA (SA), Cert Critical Care (SA); J M Brown, ${ }^{1}$ MB ChB, FCA (SA), Cert Critical Care (SA); \\ R L Mathivha, ${ }^{1}$ FC Paed (SA), Cert Critical Care (SA); I Bahemia, ${ }^{1}$ BSc Hons, Med Sci (uOM), MB ChB, DTM\&H; F Nabeemeeah, ${ }^{1}$ MB ChB; \\ N Martinson, ${ }^{2} \mathrm{MB} \mathrm{ChB}, \mathrm{DCH}, \mathrm{MFGP}, \mathrm{MD}, \mathrm{MPH}$
}

${ }^{1}$ Intensive Care Unit, Faculty of Health Sciences, University of the Witwatersrand, Johannesburg, South Africa

${ }^{2}$ Wits Health Consortium, University of the Witwatersrand, Johannesburg, South Africa

Corresponding author: S Omar (shahedicu@gmail.com)

\begin{abstract}
Background. The onset of the COVID-19 pandemic in South Africa (SA) created numerous supply challenges. Demand for diagnostic testing overwhelmed the capacity to deliver. We describe the utility and outcomes of a mobile laboratory staffed by non-laboratory healthcare workers and established to perform polymerase chain reaction (PCR) testing for the rapid diagnosis of COVID-19 at a large hospital in SA.

Objectives. To describe the performance of the mobile PCR COVID-19 laboratory. The secondary objective was to determine the prevalence of COVID-19 infections in the non-COVID intensive care unit (ICU).

Methods. This was a retrospective descriptive study of data from the newly established mobile COVID-19 PCR laboratory database and the non-COVID ICU database during the first peak of the COVID-19 pandemic (20 May - 8 August 2020) at a tertiary hospital in SA.

Results. The mobile laboratory received 1113 emergency COVID-19 PCR test requests for patients with non-COVID clinical presentations. The median (interquartile range) turnaround time was 152 (123 - 184) minutes ( $n=36)$. Primary outcome (20 May - 19 June, $\mathrm{n}=315)$ : The sensitivity and specificity were $95 \%$ and $97 \%$, respectively, and the positive and negative predictive values $82.4 \%$ and $99.2 \%$, respectively Secondary outcomes (9 June - 8 August): The prevalence of COVID-19 infections among patients admitted to the multidisciplinary adult and paediatric non-COVID ICU was $2.4 \%(n=4 / 168)$. The mean (standard deviation) COVID-19 positive rate for the mobile laboratory during this period was $18.1 \%$ (6\%). The prevalence of COVID-19 infections among medical staff in the non-COVID ICU was 3.1\% ( $n=1 / 32)$. Conclusions. The establishment of a mobile PCR laboratory staffed by non-laboratory healthcare workers during the COVID-19 pandemic provided a rapid, accurate and clinically effective solution for emergency hospital admissions with non-COVID-19 presentations.
\end{abstract}

S Afr Med J 2021;111(10):957-960. https://doi.org/10.7196/SAMJ.2021.v111i10.15690

The onset of the COVID-19 pandemic in South Africa (SA), ${ }^{[1]}$ as in Europe and North America, created numerous supply challenges, including testing for SARS-CoV-2 using reverse transcriptase polymerase chain reaction (RT-PCR). Demand for diagnostic testing overwhelmed the capacity to deliver, leading to several innovative solutions. ${ }^{[2]}$ Pooled testing was found to be an efficient and costeffective solution to the large numbers of requested tests, and the US Centers for Disease Control and Prevention released guidelines for laboratories. ${ }^{[3,4]}$ Point-of-care $(\mathrm{PoC})$ testing was suggested as another possible solution to help overcome the capacity issues faced by laboratories. ${ }^{[5]}$

In our own experience at Chris Hani Baragwanath Academic Hospital, Johannesburg, obtaining COVID-19 results from the hospital laboratory meant a waiting period of 7 - 14 days owing to a lack of onsite COVID-19 PCR testing. All emergency admissions were allocated to the COVID or non-COVID stream. Given the sheer size of our hospital ( $>2800$ beds), even a 24 -hour turnaround time was not practically acceptable in terms of safety of both patients and staff. At that early phase of the first wave of the pandemic, rapid, accurate PoC PCR testing and antigen testing were not yet available. ${ }^{[6]}$ Viral cultures are known to be time consuming, and the gold standard for the diagnosis of COVID-19 is RT-PCR, which detects SARS-CoV-2 RNA in clinical specimens. ${ }^{[7,8]}$
The university Vaccine and Infectious Disease Analytics Research Unit based at the hospital had initiated a surveillance study using PCR to test symptomatic COVID-19 patients at the hospital. However, COVID-19 may have a variable clinical presentation, with a significant proportion of patients being asymptomatic. ${ }^{[9]}$ To safely triage patients with non-COVID presentations, including trauma, acute surgical, obstetric, medical, paediatric and other emergencies, the intensive care unit (ICU) medical staff, with the assistance of a non-governmental organisation and the Perinatal HIV Research Unit (PHRU), established a mobile PCR laboratory and positioned it centrally in the hospital.

We describe the utility and outcomes of this PCR laboratory established in a mobile trailer by non-laboratory healthcare workers.

\section{Objectives}

To describe the utility and outcomes of the PCR laboratory, established in a mobile trailer and staffed by non-laboratory healthcare workers. The primary objective was to determine the clinical sensitivity, specificity, and positive and negative predictive values of the COVID19 PCR assay used for patients requiring emergency hospital resources, and the secondary objective to determine the prevalence of COVID19 infections among patients admitted to and medical staff working in the non-COVID ICU during the study period. 


\section{Methods \\ Design and setting}

This was a retrospective descriptive cross-sectional study of data from the mobile COVID-19 PCR testing laboratory database and the non-COVID ICU database during the first peak of the COVID-19 pandemic from 20 May to 8 August 2020. The study was conducted at a 2880 -bed tertiary-level academic hospital in Johannesburg, SA. Approval was obtained from the Human Research Ethics Committee (Medical) of the University of the Witwatersrand (ref. no. M201011). Informed consent was waived owing to the retrospective nature of the study and the anonymity of the data.

\section{Study population}

The study population comprised all patients requiring emergency hospital resource utilisation, from emergency surgery to ICU admission, during the study period. Objectives related to the analytical assessment of the PCR method were performed on data from the first month of operation of the mobile emergency laboratory (20 May - 19 June 2020).

\section{Emergency mobile PCR laboratory}

A trailer was donated by Gift of the Givers, together with a thermocycler (Genechecker; Genesystem, South Korea) and a 2400 SARS CoV-2 Smartchecker PCR kit (Genesystem, South Korea). There were two PCR gene targets: the $\mathrm{N}$ (nucleocapsid) gene and the RdRp (RNA-dependent RNA polymerase) gene. The PCR reactions were run for 40 cycles (CT 40). As is standard with PCR, every reaction had an internal control. Every PCR run of four tests included one positive and one negative control. In addition, we received external control materials from the National Health Laboratory Service (NHLS), and these were run at least once a month. All three external controls met the required outcomes and included positive and negative materials.

The PHRU provided three research assistants to be trained to perform the PCR tests, and the ICU team provided three clinicians who were also trained to perform the tests. Using free online applications, an electronic request form and a seamless secure electronic report that was automatically sent to the referring doctor's email were developed with support from the research unit information technology department.

Our hospital requested oversight from the NHLS. The NHLS laboratory is a South African National Accreditation System (SANAS)-accredited laboratory that complies with international standards (ISO 15189:2007). They performed the initial validation of the system prior to our consideration of its use. During this period, the only other rapid, near-patient COVID-19 PCR test validated by the NHLS was the Cepheid Xpert Xpress SARS-CoV-2. In addition, the NHLS provided both pathologist and technologist oversight. The NHLS business manager at our local facility and our hospital management facilitated the establishment of the service. The NHLS request forms and test results were submitted to the local NHLS for submission to the National Institute for Communicable Diseases, as positive results required notification of the medical condition. Technical training on the PCR equipment, test process and interpretation was provided with laboratory oversight.

\section{Data collection}

All data related to the electronic request for an emergency COVID19 PCR test were compiled from the emergency mobile laboratory database. In addition, the ICU patient database was used to compile the total number of admissions during the study period. Finally, the
ICU database was also used to compile the total medical staff and those who tested positive during the study period.

\section{Study period}

We used data from the first 30 days after initiation of the mobile laboratory, extending from 20 May 2020 to 19 June 2020, for the primary outcome. This clinical validation included 319 results. The secondary outcomes were based on a 2 -month period that included the peak of the first wave of the pandemic, extending from 9 June to 8 August. The turnaround time was calculated from one sample per run per day for 1 week during July (11 - 17 July). 9 June was selected because this was the first date of the launch of the electronic test requests enabling data availability. SA was on level 2 lockdown until 31 May and then on level 3 lockdown for the remainder of the study period.

\section{Study definitions}

Clinical validation methodology - sensitivity and specificity. For the emergency COVID-19 PCR tests performed at the mobile laboratory, there was direct communication with the referring clinicians. All clinical discrepancies were discussed directly with medical staff (mobile laboratory clinician staff).

Suspected false positives. All suspected false positives were referred to an alternative laboratory testing for two different gene targets (N1 and N2, Roche assay). This result, taken together with the clinical assessment in conjunction with the referring clinician, was considered as the clinical and laboratory gold-standard outcome.

Suspected false negatives. To detect false negatives, we used the results from an ongoing clinical study entitled 'Sentinel, hospitalbased surveillance for investigation of SARS-coronavirus-2 and other respiratory pathogens (COVID-19 study)'. This study allowed clinicians to test for COVID-19 on their inpatients when they suspected pneumonia. All positive results from this study during the evaluation period were cross-checked with the mobile laboratory tests looking for a prior negative result.

\section{Statistical analysis}

All data were assessed for normal distribution. Turnaround time had a non-parametric distribution and was described using median with interquartile range (IQR). Sensitivity, specificity and prevalence were calculated using Excel for Office 365 (Microsoft, USA) and Statistica v13 (TIBCO Software, USA).

\section{Results}

During the peak of the first wave of the pandemic (2-month period), 1113 requests were received by the mobile laboratory. Complete data were available for between 1022 (91.8\%) and 1032 (92.7\%) of the requests (Table 1). The median (IQR) turnaround time from electronic test request until secure e-mail response to referring clinician was $152(123-184)$ minutes $(n=36)$.

\section{Primary objective (20 May - 19 June 2020)}

A total of 319 tests were performed during the study period. One test was referred to another laboratory without obtaining a result from the mobile PCR system, while 3 tests were external controls and were excluded from the analysis. The total number of evaluated tests was 315 . Sample types were 281 nasopharyngeal swabs (88.1\%), 17 nasal swabs $(5.3 \%), 1$ oropharyngeal swab $(0.3 \%)$ and 7 tracheal aspirates (2.2\%); in 13 cases (4.1\%), the sample type was not stated.

Table 2 provides absolute counts of positive and negative test results. The calculated sensitivity was $95 \%$, while the specificity 
Table 1. Emergency mobile laboratory COVID-19 polymerase chain reaction requests

\begin{tabular}{llll}
\hline & All & Positive COVID-19 & Negative COVID-19 \\
\hline Age (years), mean (SD) $(n)$ & $40(20.4)\left(1032^{\star}\right)$ & $46(17.9)(185)$ & $39(20.7)(839)$ \\
Male gender, $n(\%)$ & $551(53.1)$ & $96(51.6)$ & $447(53.7)$ \\
Discipline, $n(\%)^{\dagger}$ & & & \\
General surgery & $252(24.0)$ & $40(15.9)$ & $210(83.3)$ \\
Medical & $286(28.0)$ & $81(28.3)$ & $201(70.3)$ \\
O\&G & $53(5.2)$ & $7(13.2)$ & $45(84.9)$ \\
Paediatrics & $92(9.0)$ & $17(18.5)$ & $74(80.4)$ \\
Trauma & $88(8.6)$ & $8(9.1)$ & $79(89.8)$ \\
Other & $251(24.6)$ & $31(12.4)$ & $211(84.1)$ \\
Total & $1022(100)$ & $184(18.0)$ & $820(80.2)$ \\
Reason, $n$ (\%) & & & \\
CCU admission & $27(2.6)$ & $6(22.2)$ & $21(77.8)$ \\
Emergency surgery & $270(26.3)$ & $24(8.9)$ & $244(90.4)$ \\
ICU admission & $333(32.5)$ & $63(18.9)$ & $259(778)$ \\
Other indication & $367(35.8)$ & $86(23.4)$ & $276(75.2)$ \\
Urgent IHD & $6(0.5)$ & $2(33.3)$ & $4(66.7)$ \\
Urgent transfer out & $21(2.1)$ & $4(19.0)$ & $17(81.0)$ \\
Total & $1024(100)$ & $185(18.1)$ & $821(80.2)$ \\
SD $=$ standard deviation; O\&G = obstetrics and gynaecology; CCU = cardiac care unit; ICU = intensive care unit; IHD = intermittent haemodialysis. &
\end{tabular}

Table 2. Positive and negative results and clinical outcome

\begin{tabular}{llll}
\multicolumn{1}{l}{ Table 2. Positive and negative results and clinical outcome } & COVID-19 disease present & COVID-19 disease absent & Total \\
\hline Test positive, $n$ & 42 (true positives) & 9 (false positives) & 51 \\
Test negative, $n$ & 2 (false negatives) & 262 (true negatives) & 264 \\
Total, $N$ & 44 & 271 & 315
\end{tabular}

was $97 \%$. The positive predictive value was $82.4 \%$ and the negative predictive value $99.2 \%$. The positive likelihood ratio was 32 , while the negative likelihood ratio was 0.05 .

\section{Secondary objectives}

The prevalence of COVID-19 infections among all patients admitted to the multidisciplinary adult and paediatric non-COVID ICU was $2.4 \%(n=4 / 168)$ during the study period. The contribution was as follows: $3.6 \%(n=1 / 28)$ for paediatrics, $2.9 \%(n=3 / 102)$ for adult patients, and no $(n=0 / 38)$ adult trauma patients. The mean (standard deviation) COVID-19-positive rate for the mobile laboratory during this period was $18.0 \%(6.0 \%)$. The paediatric patient was diagnosed with paediatric multisystem inflammatory syndrome and transferred out to the COVID ICU on the day of admission based on clinical suspicion. The first adult patient was a surgical patient with acute appendicitis. The patient had a repeat COVID-19 PCR test performed because of clinical suspicion and was transferred to the COVID ICU after repeat testing was positive. The second patient was an obstetric patient admitted with complications after caesarean section, who tested negative before admission to the ICU. Owing to clinical suspicion, testing was repeated at 48 hours and confirmed COVID-19 disease. She died on the same day due to respiratory complications. The last adult patient was admitted with bacterial community-acquired pneumonia after testing negative for COVID-19 immediately before ICU admission. He improved and was extubated. However, on day 4 of his ICU stay, he was retested on the basis of clinical suspicion.
He tested positive and was transferred to the COVID medical ward as he did not require intensive care.

The prevalence of COVID-19 infections among medical staff working in the non-COVID ICU during the study period was $3.1 \%$ $(n=1 / 32)$.

\section{Discussion}

The main finding of our study was that we were able to establish a sensitive and specific COVID-19 PCR laboratory using a mobile trailer and non-laboratory healthcare workers during the first wave of the COVID-19 pandemic. Our mobile PCR platform provided a sensitivity of $95 \%$ and a specificity of $97 \%$. A systematic review and meta-analysis by Floriano et al. ${ }^{[10]}$ pooled data from 13 studies and showed a sensitivity of $86 \%(84-88 \%)$ and specificity of $96 \%$ (94 $97 \%$ ). There was a low risk of bias, and $>6000$ patients were included. However, this study was based on PCR performed for coronavirus excluding SARS-CoV-2. Data from our study for SARS-CoV-2 are in keeping with these findings. A more recent systematic review found a sensitivity range of $71-98 \%$ for SARS-CoV-2 PCR testing. ${ }^{[11]}$

Looking specifically at the false-negative rates, we found only $2 / 264$ false negatives $(<1 \%)$. Our definition of false negatives is based on the finding of clinical pneumonia after an initial negative test. Using repeat testing, this figure has been shown to be as high as $29 \% .{ }^{[12]}$ More recently, Zhang et al. ${ }^{[13]}$ applied an innovative strategy using serological testing and found a false-negative rate for PCR of $4.5 \%$. They found that $92 \%$ of seropositive contacts of known positive patients who were PCR negative earlier reported no symptoms. 
A potential relationship between viral shedding and symptoms can be postulated ${ }^{[14,15]}$ and may explain the low false-negative rate in our study, since we could only search for pneumonia cases (symptomatic disease) in our PCR-negative group.

No diagnostic test for COVID-19 is 100\% accurate, and apart from the issue of false negatives, false-positive results may also have significant consequences. ${ }^{[16]}$ The false-positive rate in our study was $3.3 \%$. This figure compares well with rates reported by others, ranging between $0.8 \%$ and $4 \% \cdot{ }^{[17]}$ The impact of false-positive results ranges from health-related consequences such as cancellation of elective surgery or diversion to an incorrect hospital stream to global consequences such as overestimation of disease burden. ${ }^{[16]}$

The prevalence of nosocomial COVID-19 infections among ICU admissions in our study was $2.4 \%(n=4 / 168)$. This was in the setting of PCR testing on the day of admission using the emergency laboratory service. A turnaround time of $\sim 150$ minutes was found to be a reasonably practical period to avoid bottlenecks in the system. A meta-analysis by Zhou et al. ${ }^{[18]}$ that included 40 studies revealed a nosocomial infection rate of $44 \%$ for COVID-19. Data from the same meta-analysis showed that both severe acute respiratory syndrome (SARS) and Middle East respiratory syndrome (MERS) had similarly high rates of $36 \%$ and $56 \%$, respectively. These figures suggest that the mobile laboratory, which processed $>1000$ samples during the study period, may have had a significant role in limiting the nosocomial spread of COVID-19. Rhee et al. ${ }^{[19]}$ demonstrated in a single academic hospital in the USA that the use of a rigorous infection control programme that included personal protective equipment, liberal access to PCR testing, and separation of COVID-19 and nonCOVID-19 patients, among other strategies, was able to achieve a nosocomial infection rate of $7.6 \%(n=697 / 9149)$. Data from this large study support our findings.

Looking at the ICU medical staff, the prevalence of hospitalacquired COVID-19 infections during the study period was 3.1\% $(n=1 / 32)$. In the study by Zhou et al. ${ }^{[18]} 33 \%$ of the $44 \%$ of nosocomial infections affected medical staff, resulting in an infection rate of $15 \%$ for this group. It may be postulated that the mobile laboratory had a significant contributory role in preventing nosocomial COVID-19 infections among medical staff.

With appropriate training, we were able to use non-laboratory personnel to perform all the laboratory functions required for COVID-19 PCR testing. Laney et al. ${ }^{[20]}$ have shown that, given appropriate training, non-medical, non-healthcare laypeople using diagnostic tests can achieve similar accuracy to trained laboratory personnel. We were able to show that during a disaster situation (COVID-19 pandemic), flexible and innovative utilisation of healthcare resources, including healthcare workers, can alleviate critical bottlenecks, resulting in efficient processes with positive outcomes.

\section{Study limitations}

The retrospective nature of the study and the relatively small number of important outcomes are limitations. However, our findings are consistent with the outcomes of larger studies.

\section{Conclusions}

The establishment of a mobile PCR laboratory staffed by nonlaboratory healthcare workers during the COVID-19 pandemic provided a rapid, accurate and clinically effective solution for emergency hospital admissions with non-COVID-19 presentations.

\section{Declaration. None.}

Acknowledgements. The authors wish to thank Dr Imtiaz Sooliman of the Gift of the Givers Foundation for funding the mobile laboratory trailer, equipment and consumables throughout the first wave of the COVID-19 pandemic.

Author contributions. All authors made substantial contributions to all of the following: the conception and design of the study, or acquisition of data, or analysis and interpretation of data; drafting the article and revising it critically; and approving the final version to be submitted.

Funding. None.

Conflicts of interest. None.

1. National Institute for Communicable Diseases. First case of COVID-19 coronavirus reported in SA. 5 March 2020. https://www.nicd.ac.za/first-case-of-covid-19-coronavirus-reported-in-sa/ (accessed 23 February 2021)

2. Fadal R, Wadee S, Hoosen A, Parker W. Maximising the efficiency of surveillance for COVID-19 in dialysis units in South Africa: The case for pooled testing. S Afr Med J 2020;110(8):707. https://doi. org/10.7196/SAMJ.2020.v110i8.15008

3. Iacobucci G. Covid-19: Study findings strongly support use of pooled testing, say researchers. BMJ 2020;3:371. https://doi.org/10.1136/bmj.m4267

4. Centers for Disease Control and Prevention. Guidance for SARS-CoV-2 point-of-care and rapid Centers for Disease Control and Prevention. Guidance for SARS-CoV-2 point-of-care and rapid
testing. Revised 8 March 2021. https://www.cdc.gov/coronavirus/2019-ncov/lab/point-of-care-testing. html (accessed 12 April 2021).

$\mathrm{html}$ (accessed 12 April 2021).
5orld Health Organization. Advice on the use of point-of-care immunodiagnostic tests for COVID-19. 8 April 2020. https://www.who.int/news-room/commentaries/detail/advice-on-the-useof-point-of-care-immunodiagnostic-tests-for-covid-19 (accessed 12 April 2021).

6. Riccò M, Ferraro P, Gualerzi G, et al. Point-of-care diagnostic tests for detecting SARS-CoV-2 antibodies: A systematic review and meta-analysis of real-world data. J Clin Med 2020;9(5). https:// doi.org/ $10.3390 / \mathrm{jcm} 9051515$

7. Mitchell SL, St George K, Rhoads DD, et al. Understanding, verifying, and implementing emergency use authorisation molecular diagnostics for the detection of SARS-CoV-2 RNA. J Clin Microbiol 2020;58(8):e00796-20. https://doi.org/10.1128/icm.00796-20

8. Tang Y-W, Schmitz JE, Persing DH, Stratton CW. Laboratory diagnosis of COVID-19: Current issues 8. Tang Y-W, Schmitz JE, Persing DH, Stratton CW. Laboratory diagnosis of COVID-19: Curren
and challenges. J Clin Microbiol 2020;58(6):e00512-20. https://oi.org/10.1128/jcm.00512-20

9. Tabata S, Imai K, Kawano S, et al. Clinical characteristics of COVID-19 in 104 people with SARS$\mathrm{CoV}-2$ infection on the Diamond Princess cruise ship: A retrospective analysis. Lancet Infect Dis 2020;20(9):1043-1050. https://doi.org/10.1016/S1473-3099(20)30482-5

10. Floriano I, Silvinato A, Bernardo WM, et al. Accuracy of the polymerase chain reaction (PCR) test in the diagnosis of acute respiratory syndrome due to coronavirus: A systematic review and metaanalysis. Rev Assoc Med Bras 2020;66(7):880-888. https://doi.org/10.1590/1806-9282.66.7.880

11. Arevalo-Rodriguez I, Buitrago-Garcia D, Simancas-Racines D, et al. False-negative results of initial RT-PCR assays for COVID-19: A systematic review. medRxiv 2020 (epub 13 August 2020). https://doi. RT-PCR assays for COVID-19: A s

12. Watson J, Whiting PF, Brush JE. Interpreting a covid-19 test result. BMJ 2020;369:m1808. https://doi. org/10.1136/bmj.m1808

13. Zhang Z, Bi Q, Fang S, et al. Insight into the practical performance of RT-PCR testing for SARS-CoV-2 using serological data: A cohort study. Lancet Microbe 2021;2(2):e79-e87. https://doi.org/10.1016/ s2666-5247(20)30200-7

14. Kucirka LM, Lauer SA, Laeyendecker O, Boon D, Lessler J. Variation in false-negative rate of reverse transcriptase polymerase chain reaction-based SARS-CoV-2 tests by time since exposure. Ann Intern Med 2020;173(4):262-267. https://doi.org/10.7326/M20-1495

15. He X, Lau EHY, Wu P, et al. Temporal dynamics in viral shedding and transmissibility of COVID-19. Nat Med 2020;26(5):672-675. https://doi.org/10.1038/s41591-020-0869-5

16. Surkova E, Nikolayevskyy V, Drobniewski F. False-positive COVID-19 results: Hidden problems and costs. Lancet Respir Med 2020;8(12):1167-1168. https://doi.org/10.1016/s2213-2600(20)30453-7

17. Cohen AN, Kessel B, Milgroom MG. Diagnosing SARS-CoV-2 infection: The danger of over-reliance on positive test results. medRxiv 2020 (epub 28 September 2020). https://doi. org/10.1101/2020.04.26.20080911

18. Zhou Q, Gao Y, Wang X, et al. Nosocomial infections among patients with COVID-19, SARS and MERS: A rapid review and meta-analysis. Ann Transl Med 2020;8(10). https://doi.org/10.21037/atm20-3324

19. Rhee C, Baker M, Vaidya V, et al. Incidence of nosocomial COVID-19 in patients hospitalised at a large US academic medical center. JAMA Netw Open 2020;3(9):e2020498. https://doi.org/10.1001/ jamanetworkopen.2020.20498

20. Laney W, Mawelele R, Omar S. The accuracy of a point-of-care test among different operators using the QBC Autoread Plus Analyzer for the measurement of a basic full blood count. S Afr Med J 2019;109(12):952-956. https://doi.org/10.7196/SAMJ.2019.v109i12.13981

Accepted 26 May 2021. 